\title{
A PILOT STUDY ON USING ACUPUNCTURE AND CORE STABILITY EXERCISES TO TREAT NON-SPECIFIC ACUTE LOW BACK PAIN AMONG INDUSTRIAL WORKERS
}

\begin{abstract} department in the UK. stability exercise group (CSG). three-months follow up.

\section{INTRODUCTION}

Back pain in industry is a major problem. The prevalence of LBP in the working population varies from one country to another depending on the profession. Ghaffari et al. (2006.) in Iran carried out a cross- sectional design study involving 1400 industrial workers, and reported
\end{abstract}

OBJECTIVE: To explore the effects of using acupuncture and core stability exercises to treat acute low back pain among industrial workers at an Occupational health

METHODS: Consecutive samples of patients with acute low back pain, $L B P(n=15)$ of less than 12 weeks duration, who attended the Physiotherapy Department at the Occupational Health Unit during the study were recruited. Subjects were randomly allocated to one of the three groups (using a computer-generated table of randomisation), acupuncture group (ACG), acupuncture and core stability exercise (EACG) and core

$A C G$ received acupuncture treatment at selected points, for low back pain. CSG received instruction on core stability muscle activation, while the EACG received a combination of acupuncture and core stability exercise treatment. Each group received treatment once weekly for six weeks during the study. Visual analogue scale (VAS), Rolland and Morris Disability Questionnaire (RMDQ), and medication use were completed at baseline, at the end of six-week treatment and at

RESULTS: All the groups reported lower VAS and RMDQ scores at the end of the treatment and at three-months follow up. However, significant reduction in pain intensity $(P<0.008)$ and significant reduction in $R M D Q$ scores (improvement in $Q O L)$ were shown in the EACG. The majority of the patients in the EACG were taking fewer medications at the end of the treatment, with further progression to no medication intake at the three-month follow up.

CONCLUSION: The study demonstrated that combined acupuncture and core stability exercise treatment are effective for pain relief among industrial workers, reduction of back pain, functional disability and pain medication intake in patients suffering from acute low back pain for up to three months post-intervention.

\section{KEYWORDS: LBP, ACUPUNCTURE, EXERCISE, CORE STABILITY, PAIN}

\section{Corresponding author:}

Sokunbi OG

Department of Medical Rehabilitation, College of Medical Sciences University of Maiduguri

E-mail:ganiyusokunbi@gmail.com one-year prevalence of $20 \%$ and $27 \%$ for male and female workers respectively. In Sweden, Linton et al. (2011) reported one-year prevalence of $63 \%$ and $69 \%$ for males and females in a population-based survey comprising 2400 workers. In a general working population in Sweden aged 20-59 years, approximately 5\% sought care because of a new LBP episode during a three-year period (Vingard 2004). In a study of LBP among scaffolders, $34 \%$ of the population had been on sick leave for at least one episode of low back pain during a three-year follow up study (Elders and Bradford 2004).

Low back disorder constitutes one of the common diagnoses behind longterm absence, sickness and disability in Western countries (Vingard et al. 2004). Although back disorders are the common cause of sick leave, not all back disorders lead to sickness. Many occupations involve tasks that LBP sufferers can perform at least to some extent, and in such a case it is not always advisable to take sick leave (Vingard et al. 2004). Workers who take time off are, by implication, believing that the work is likely to do further harm, and that a long period of absence from work would help in the resolution of their symptoms, thus taking unnecessary sickness absence or 
even losing their jobs. On the contrary, prolonged absence from work could increase the risk of chronic pain, psychological distress and consequent financial and family stress (Vingard et al. 2004). It has been suggested that almost half the number of acute back episodes will clear within one month (Langevin and Sherman 2007). It is therefore important to begin therapy at a time when the patients are still in pain. It is only then that they can appreciate the effects of treatment on their condition (Di Fabio et al, 1995). Suitably qualified physiotherapists are in a good position to provide these treatments in an on-site capacity where the employee can be seen when their pain begins.

The extent of cost to industry and the apparent difficulty in managing back pain has led to a number of prevention initiatives. The majority of preventive measures in industry are aimed at reducing the impact of physical working conditions on the back, by ergonomic adjustments, instructions on lifting techniques and lumbar support (Ghaffari et al 2006), or increasing the strength of the back muscles and general fitness (Hasegewa 2013).

On a global scale, it is of concern that while both ergonomic and education approaches have been available for some time, work absence through LBP continues to soar (Elders and Bradford 2004). With the current level of understanding and knowledge of acute back pain, it appears that management of this pain among industrial workers should not focus on the primary prevention alone, but also on consideration of an effective treatment programme. Clinical guidelines recommend advice and medication as routine care for acute back pain (Rosen 1994). Conservative interventions such as physiotherapy and ergonomic adjustment might play a major role in the treatment of acute and chronic back disorders among industrial workers. Therefore there is a need to determine whether conservative interventions have a significant impact on short, medium and long-term outcomes.

The spinal stabilisation exercise model is an active approach to LBP based on a programme of motor control exercises. The main aim of this programme is to re-establish the impairment or deficit in motor control around the neutral zone of the spinal motion segment, by restoring the normal function of the local stabiliser muscles (Costa et al. 2009). The stabilisation exercise programme has become the most popular treatment method in spinal rehabilitation since it has shown its effectiveness in some aspects related to pain and disability (Sokunbi et al. 2002). However, some studies have reported that the specific stabilisation exercise programme reduces pain and disability in chronic, but not acute low back pain. However, it can be helpful in the treatment of acute LBP by reducing recurrence rate (Franca et al. 2011). Stabilisation exercises have become a major focus in spinal rehabilitation as well as in prophylactic care such as injury prevention (Zazulak et al. 2008); the therapeutic evidence in terms of postural control variables has not been well documented. Some randomised control trials have comprehensively reported the effects of core stability exercises versus conventional physiotherapy treatment regimes on pain characteristics, recurrence and disability scores in chronic LBP patients, emphasising patient-centred outcomes (Dankaerts et al. 2006; Liddle et al. 2007). These studies have addressed the need of the homogeneous chronic LBP group for better clinical outcomes. Evaluating postural control parameters such as centre of pressure displacements, moments and forces following interventions, particularly stability exercises, may provide insight into how these surrogate outcomes are mediated by different subgroups or heterogeneous chronic LBP patients. and identifying subgroups of chronic LBP patients who are most likely to benefit from particular intervention (Muthukrishnan et al. 2010).

Both acupuncture and exercises have been reported to have significant effects on pain reduction and improving

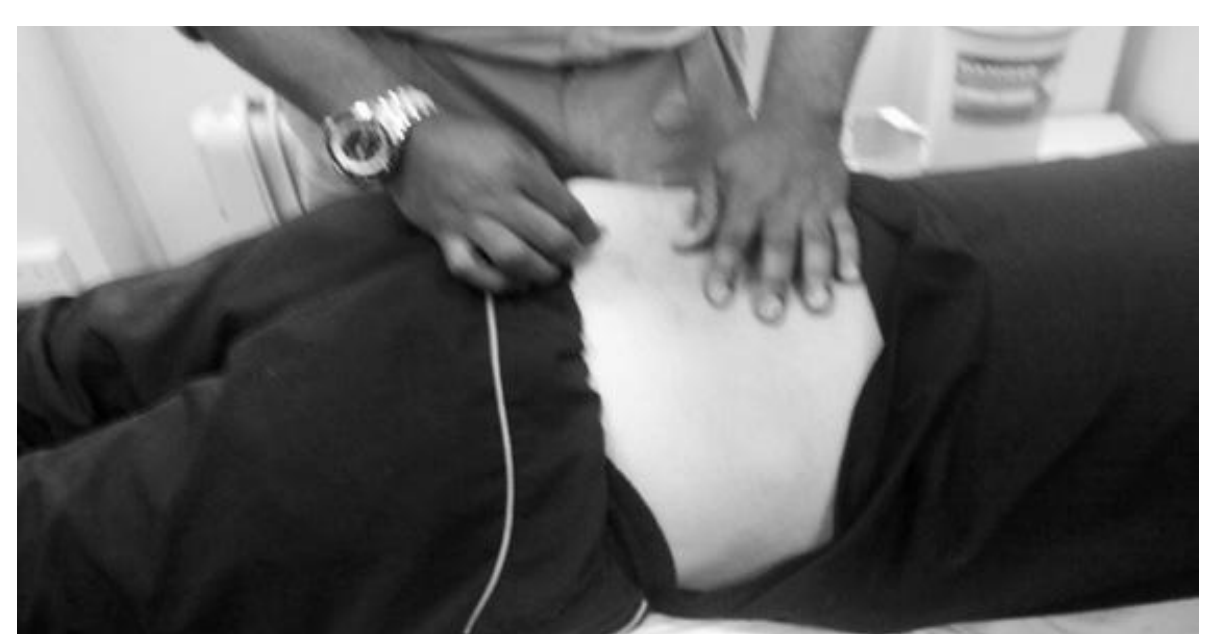

Figure 1: Clinician administering acupuncture treatment on patient lower back area

quality of life among back pain sufferers (Brinkhaus et al. 2006), but there remains insufficient evidence of its efficacy, and more research is needed to evaluate its short, medium and longterm effects among industrial workers who suffer from acute LBP. The present study aimed at testing the effects of acupuncture and core stability exercises on acute LBP among industrial workers.

\section{METHODS \\ Subjects}

Patients who were on the waiting list for physiotherapy treatment through referral from their GP were contacted by telephone and screened for inclusion in the study. Fifteen consecutive patients ( 9 males, 6 females) with acute LBP among workers from Cummins Engine, Daventry, were randomly allocated into groups using a computer-generated randomisation table (htpp:/www.randomization.com/). To ensure concealment of allocation, a member of staff not otherwise involved in the study held the randomisation list.

\section{Inclusion criteria}

Males and females aged 18-65 years who were able to give informed consent were included in this study. Other criteria for participation included a current episode of non-specific LBP (not more than 12 weeks), with or without referred pain. LBP is defined as pain, muscle tension, stiffness localised below the costal margin and above inferior gluteal fold. Patients on drug treatment were included if there had been no change in medicine and its dosage for one month or longer.

\section{Exclusion criteria:}

The exclusion criteria were major trauma or systemic diseases, generalised dermopathologies, pregnancy, inability or unwill- 
ingness to answer any of the questions required to complete the questionnaires, and conflicting ongoing interventions. Prior experience of acupuncture treatment and core stability treatment were considered as exclusion criteria to this study.

\section{Ethical consideration}

Approval to carry out this study was obtained from the Cummins, Daventry Research Ethical Committee. Subjects personally received information about the procedures of the study and were enrolled after giving their informed consent.

According to the randomisation table, consecutive samples of subjects were allocated to groups, namely EACG, ACG and CSG. Evaluation of the patients assigned to the three intervention groups for the inclusion and exclusion criteria and for outcome measures was carried out by a professional who was blinded to the assignment of the different treatment options.

\section{Study intervention}

Acupuncture treatment and core stability muscle exercise training were carried out by a specialist physiotherapist who was a certified acupuncturist (by Acupuncture Association of Chartered Physiotherapist) and also had 16 years of clinical experience of physiotherapy practice of using core stability exercises in the management of low back disorders.

\section{Acupuncture group (ACG)}

The ACG patients received acupuncture treatment at selected acupuncture points for 20 minutes on the affected low back pain area. The selected acupuncture points used in the present study are widely accepted for treating LBP (Molsberger et al. 2002), namely Shensu (BL23), Dachangshu (BL25), Ciliao (BL32), Weizhong (BL40), Kunlum (BL60), Huantiao (GB30) and Yanglingquan (GB34). Disposable stainless steel needles $(0.2 \mathrm{mmx} 40 \mathrm{~mm}$, Seirin co Ltd) were inserted into a muscle to a depth of $10 \mathrm{~mm}$ using sparrow pecking acupuncture technique (alternate pushing and pulling of the needle). The needle manipulation was stopped when the subject felt dull pain or acupuncture sensation (numbness, soreness and or radiating sensation) and the needle was left in position for another 20 minutes (Figure 1).

\section{Core stability exercise group (CSG)}

The researcher individually showed the subjects the location of core stabil-

Figure 2: Study flow chart

\section{Individuals assessed for eligibility}

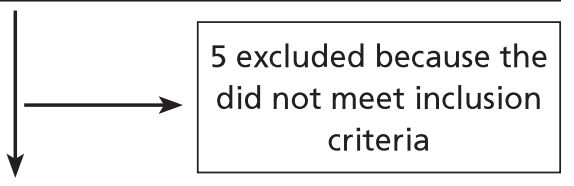

15 randomised to participate in the study

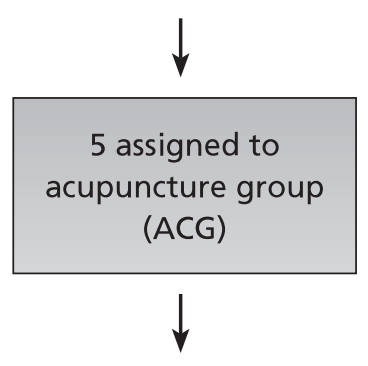

$$
\begin{aligned}
& 5 \text { completed } 6 \text { week } \\
& \text { treatment and } 3 \\
& \text { month follow up }
\end{aligned}
$$
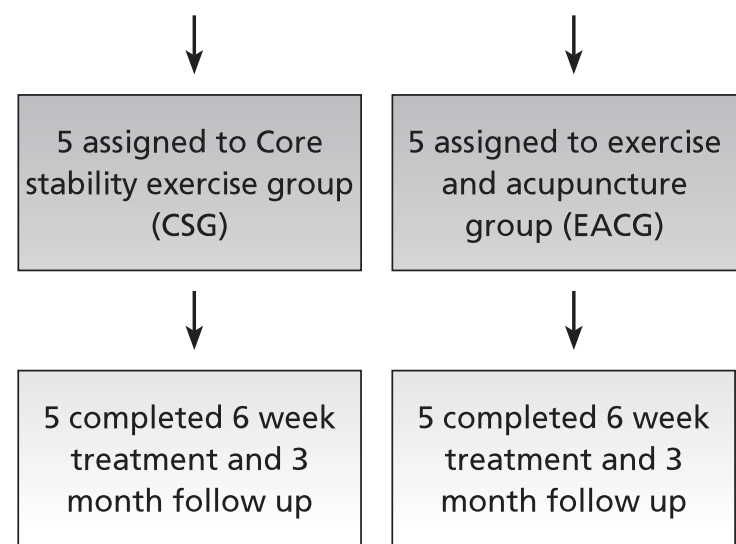

ity muscle in the body and how to activate the main core stability muscles. The techniques of core stability muscle activation used in this study were as described by Sokunbi et al. (2002). Core stability exercises were carried out for 20 minutes.

\section{Exercise and Acupuncture group (EACG)}

Subjects in the EACG combined the treatment protocols for ACG and CSG. The patients received 20 minutes' treatment of acupuncture and 20 minutes of treatment with core stability muscle exercises.

\section{Blinding}

Given the nature of the treatments, it was not possible to blind subjects and the therapist with respect to the content of the interventions. An investigator who was not aware of patient allocation until the completion of data analysis carried out data collection at all time points.

\section{Outcome measures}

Visual Analogue Scale (VAS, 0-100mm) was used to measure pain intensity. Rolland and Morris Disability questionnaire (RMDQ) was used to measure disability due to pain (von Korff et al. 2000). The VAS and RMDQ scores were measured immediately before the first treatment, at the end of the six-week treatment, and at three months follow up. Medication intake (number of tablets taken per day) was used as the secondary outcome measure, which was also recorded at baseline after six weeks' treatment and at three months follow up.

\section{Analysis}

All variables were analysed using Microsoft Excel (2010) by an investigator who was masked to treatment allocation. Repeated analysis of variance was used to study the changes in VAS and RMDQ scores in the three groups. After detection of significant changes in the overall time course with ANOVA, post-hoc analysis was carried out, using Bonferroni correction. Changes among and within groups were considered significant at a level of $\mathrm{P}<0.008$

\section{RESULTS}

The flow chart from the beginning of the study, with 20 individuals assessed for eligibility is illustrated in figure 2. Five subjects were excluded because they did not meet the inclusion criteria. Fifteen patients were finally randomised into three groups of five subjects each. None of the subjects in all three groups withdrew before the end of the study (three months follow up).

Table 1: shows the baseline characteristics of the participants. There were no statistically significant differences in the average age of participants among the 


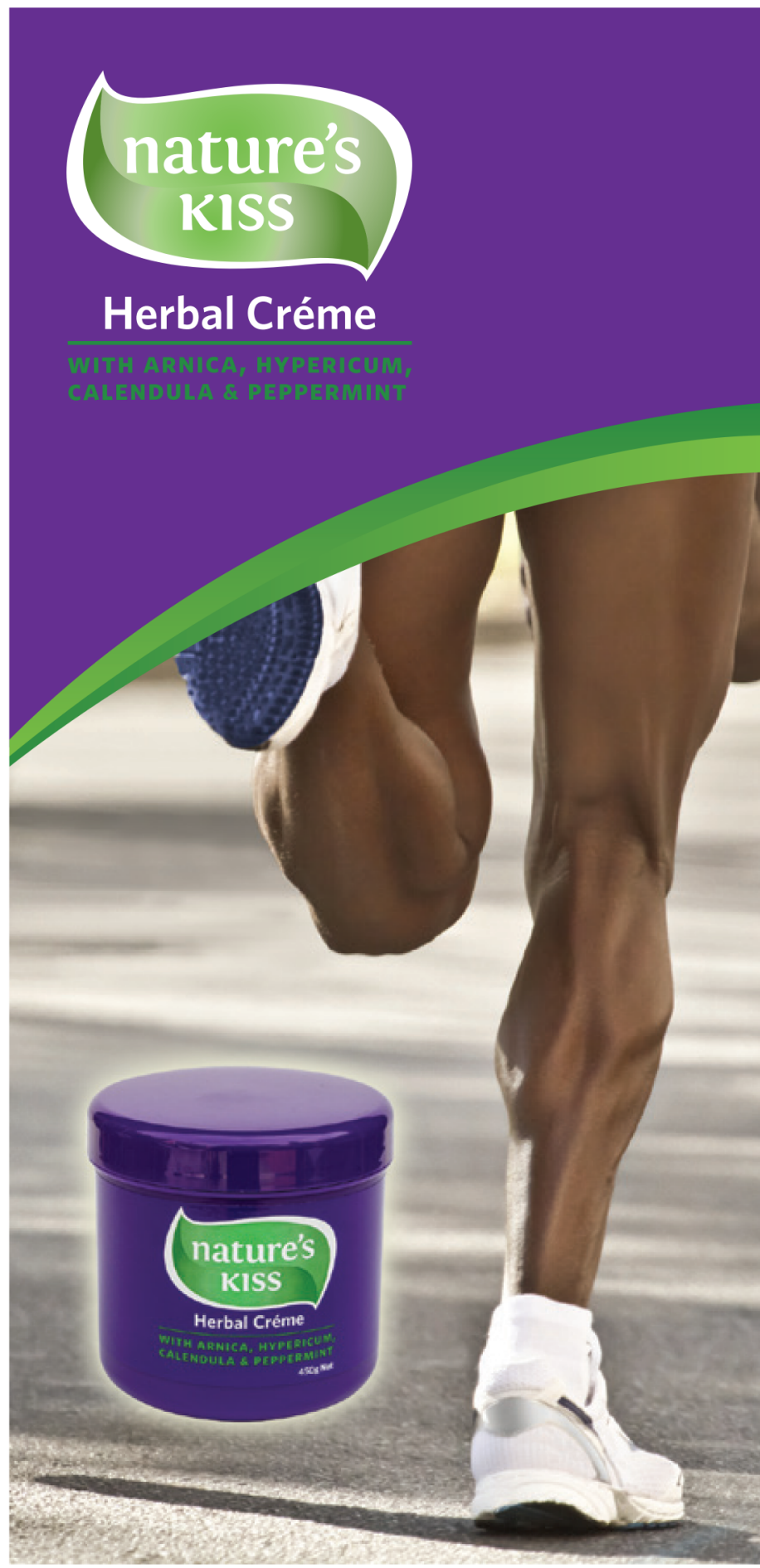

Available and recommended by physiotherapists, massage therapists, health shops and pharmacies

CONTACT US

\section{Frieda Bernard Consultants} trading as Nature's Kiss

0114861622 / 0114862722 romyb@global.co.za / cfulton@global.co.za

www.natureskiss.co.za three groups $(\mathrm{P}=0.059)$. The majority of the participants ( 12 , $80 \%$ ) experienced pain localised in the lower back region only, while the remaining $20 \%$ suffered from referred pain in the lower extremity. Many of the participants (13, 86.7\%) were taking one form or another of analgesics at the start of the treatment.

\section{Pain intensity (VAS) scores}

Table 2: The mean pain intensity scores decreased in the entire group at the end of the six-week treatment although the extent varied among the group. In the EACG, the pain intensity score was significantly lower at the end of the sixweek treatment, and at the three-month follow up $(\mathrm{P}<0.08)$. The CSG group recorded a significantly lower pain intensity score at the three-month follow up $(\mathrm{P}<0.008)$ which was not experienced after the six-week treatment $(\mathrm{P}=0.07)$.

\section{RMDQ Scores}

Table 3: The mean RMDQ scores decreased in the entire group at the end of the six-week treatment although the extent varied among the group members. Disability due to low back pain was significantly reduced at the three-month follow up $(\mathrm{P}<0.008)$. The EACG recorded significantly lower RMDQ scores after the six weeks of treatment and at the three-month follow up $(\mathrm{P}<0.008)$.

Table 4: The majority of the patients $(86.7 \%)$ were taking medication at the start of the treatment. At the end of the six-week treatment, the EACG was taking a smaller number of medications, with further progression to no medication intake at follow up.

\section{DISCUSSION}

The results of this study demonstrated that acupuncture combined with core stability muscle exercises is more effective than either acupuncture treatment or core stability exercise treatment alone. The study also demonstrated that a combination of acupuncture and core stability exercise treatment could have both short-term and medium-term benefits.

A stabilisation exercise program has been shown to produce short-term improvements in global impression of recovery and activity for people with low back pain, maintaining the results after six and twelve months (Costa et al.. 2009), as well as being superior to minimal intervention at long-term follow-up (Macedo et al.. 2009; Kriese 2010). Improvements in pain intensity and functional disability were also demonstrated in groups of patients with low back pain suffering from a spondylolisis, or a spondylolisthesis and a significant decrease of symptoms in people with hypermobility (Pereira et al. 2011). Studies on the effects of stabilisation exercises on back pain among industrial workers appeared to be scarce.

Acupuncture analgesia improved the noxious descending inhibitory controls and pain gate mechanism, and therefore helped the patients' pain levels. The overall result was that of pain reduction, reduction in functional disability due to pain, and improving wellbeing. Like other studies (Leibing et al. 2002) it is difficult to ascertain to what extent acupuncture has helped these patients, as psychological components could not be measured. Positive reinforcement might co-exist in acupuncture and core stability exercises, and psychological components could very well be a positive element in getting better. A preliminary paper by Gamus et al. (2008) demon- 
strates that pain perception and coping strategies can be positively affected by acupuncture, although it is not yet clear how the limbic system, somatosensory cortex, brainstem, cerebellum can be stimulated (Gamus et al. 2008).

The findings in the present study are analogous to those of previous studies involving both acute and chronic LBP patients (Furlan et al. 2005, Manhemer et al. 2005, vanTudler et al. 2000). Taking this information into account, it could be suggested that a future larger study focusing on the effects of acupuncture and core stability exercise treatments would be useful in establishing whether or not such a combination of treatment techniques would have an effect in reducing the rate and intensity of recurrent pain, reducing the risk of chronic disability, and reducing work absenteeism.

\section{LIMITATION}

Apart from the study being underpowered by the small numbers of subjects, a limitation of the study was the record of medication intake. Patients were asked to record in a daily diary the type of medication, the dose in $\mathrm{mg}$ and the number taken per day, but the compliance was poor with respect to the type of medication and dosage taken. Therefore only the number of tablets taken per day was used in the final analysis. In future studies, it will be more useful to record detailed medication intake prior to each treatment and/or to provide patients with a prompt for a daily entry (either by telephone or electronically).

\section{CONCLUSION}

This study has demonstrated that combined acupuncture and core stability exercise treatment are effective for pain relief among industrial workers, reduction of back pain, functional disability and pain medication intake in patients suffering from acute low back pain for up to three months post-intervention. A large-scale randomised clinical trial is warranted.
Table 1: Baseline characteristics of participants

\begin{tabular}{|c|c|c|c|c|}
\hline Characteristics & $\begin{array}{l}\text { ACG } \\
(n=5)\end{array}$ & CSG $(n=5)$ & $\begin{array}{l}\text { EACG } \\
(n=5)\end{array}$ & Statistics \\
\hline Age $X(S D)$ & $40.3(8.2)$ & $42.1(9.3)$ & $41.4(10.3)$ & $\begin{array}{l}F=3.885 \\
P=0.059\end{array}$ \\
\hline \multicolumn{5}{|l|}{ Sex } \\
\hline M & $3(60 \%)$ & $2(40 \%)$ & $4(80 \%)$ & \\
\hline $\mathrm{F}$ & $2(40 \%)$ & $3(60 \%)$ & $1(20 \%)$ & \\
\hline \multicolumn{5}{|l|}{ Areas of pain } \\
\hline Back pain only & $4(80 \%)$ & $5(100 \%)$ & $3(60 \%)$ & \\
\hline Back and leg pain & $1(20 \%)$ & 0 & $2(40 \%)$ & \\
\hline \multicolumn{5}{|l|}{ Medications } \\
\hline Diclofenac & $1(20 \%)$ & 0 & $1(20 \%)$ & \\
\hline Ibuprofen & $2(40 \%)$ & $2(40 \%)$ & $2(40 \%)$ & \\
\hline Paracetamol & $1(20 \%)$ & $3(60 \%)$ & $2(40 \%)$ & \\
\hline No medication & $1(20 \%)$ & 0 & 0 & \\
\hline Other medications & 0 & 0 & 0 & \\
\hline
\end{tabular}

Table 2: Pain intensity scores (VAS)

\begin{tabular}{|l|l|l|l|}
\hline VAS SCORES & ACG $(n=5)$ & CSG $(n=5)$ & EACG $(n=5)$ \\
\hline & X(SD) & X(SD) & X(SD) \\
\hline Baseline & $62.8(15.7)$ & $60.0(18.5)$ & $62.7(17.8)$ \\
\hline 6 week of treatment & $59.5(20.5)$ & $53.3(10.5)$ & $40.8(3.7)$ * \\
\hline 3 months follow up & $58.0(22.5)$ & $50.9(10.4) *$ & $36.6(5.3)$ * \\
\hline * significant $(P<0.08)$ &
\end{tabular}

Table 3: Roland Morris Disability Questionnaire (RMDQ) scores

\begin{tabular}{|l|l|l|l|}
\hline RMDQ SCORES & ACG $(n=5)$ & CSG $(n=5)$ & EACG $(n=5)$ \\
\hline & $X(S D)$ & $X(S D)$ & $X(S D)$ \\
\hline Baseline & $8.2(3.7)$ & $8.0(3.1)$ & $9.0(4.5)$ \\
\hline 6 week of treatment & $6.5(3.5)$ & $6.4(3.1)$ & $3.8(0.7)$ * \\
\hline 3 months follow up & $7.5(2.6)$ & $4.1(2.4) *$ & $3.3(0.3)$ * \\
\hline * significant $(P<0.08)$ & &
\end{tabular}

Table 4: Daily Medication intake (Number of tablets)

\begin{tabular}{|l|l|l|l|}
\hline NUMBER OF TABLETS & ACG $(n=5)$ & CSG $(n=5)$ & EACG $(n=5)$ \\
\hline & $X(S D)$ & $X(S D)$ & $X(S D)$ \\
\hline Baseline & $12.0(1.5)$ & $12.4(2.3)$ & $10.9(2.2)$ \\
\hline 6 week of treatment & $10.5(2.3)$ & $10.4(2.9)$ & $4.5(1.3) *$ \\
\hline 3 months follow up & $8.9(2.4)$ & $4.8(0.9) *$ & 0.0 \\
\hline * significant $(P<0.08)$ & & \\
\hline
\end{tabular}




\section{REFERENCES}

Brinkhaus B, Witt CM, Jena S, Linde K, Streng A, Wagenpfeil S, Irnich D, Walther H-U, Melchart D, Willich SN 2006. Acupuncture in patients with chronic low back pain. Archives of Internal Medicine 166:450-457.

Costa LOP, Maher CG, Latimer J,1 2009. Motor control exercise for chronic low back pain: a randomized placebo-controlled trial. Physical Therapy; 89: 1275-86.

Dankaerts W, O'Sullivan P, Burnett, A, Straker L 2006. Altered patterns of superficial trunk muscle activation during sitting in nonspecific chronic low back pain patients: importance of subclassification. Spine; 31(17): 2017-23

Di Fabio, R., P., Mackey, G., and Holt, J.B. 1995. Disability and functional status in patient with low back pain receiving workers compensation. A descriptive study with implication for the efficacy of physical therapy: Physical Therapy 75, 3 180-19

Dixon, A. 1976; Diagnosis of low back pain In Jayson, M(ed). The Lumabr spine and Back pain, Pitman publishing. Tunbrdige wells

Elders L.A., Burdford A, 2004 Prevalence, incidence and reccurence of low back pain in scaffolders in a 3 year follow up study Spine 6: 101-106

Feyer, A.,M., Williamson A., Mandryk, J., de Silva, I., and Healy, S., 1992. The role of psychosocial risk factors in work related low back pain. Scandinavian Journal of Work, Environment and Health, 18, 368375

França FR, Burke TN, Hanada ES, Marques AP 2011. Segmental stabilization and muscular strengthening in chronic low back pain: a comparative study. Journal of Manipulative Physiol Therapy; 34(2): 98-106

Furlan, A.D., van Tudler, M.W., Cherkin, D., Tsukayama, H, Lao, L., Koes, B., Berman, B., 2005 Acupuncture and dry needling for low back pain. Cochrane Database Systematic Review: CD001351

Gaffarri M, Lipour A, Jensen I, Faeshad AA Vingard EE Low back pain among Iranian industrial workers Occupational Medicine 2006;56 :455- 460

Gamus D, Meshulam-Atzmon V, Pintov S, Jacoby R 2008. The Effect of Acupuncture Therapy on Pain Perception and Coping Strategies: A Preliminary Report. Acupuncture Meridian Studies; 1:51-53.
Hasegawa TM, Baptista AS, de Souza MC, Yoshizumi AM, Natour J. 2013. Acupuncture for acute non-specific low back pain: a randomised, controlled, double-blind, placebo trial. Acupuncture Medicine.10.1136

Hildebrandt, V.H., 1995. Back pain in the working population: prevalence, rates in Dutch trades and professions Ergonomics 38 :1283-1298

Hyytiainen, K 1994. Attitudes towards prevention of low back disorders in industry: Occupational Medicine 44:83-86

Kriese M, Clijsen R, Taeymans J, Cabri J.200 Segmental stabilization in low back pain: a systematic review. Sportverletz Sportschaden 24(1): $17-25$

Lahad, A., Malter, A.D., Berg A.O., and Deyo R.A 1996. The effectiveness of four interventions for the prevention of low back pain 1994. J. Am. Med. Assoc. 272: 1286-129

Langevin HM and Sherman KJ 2007. Pathophysiological model for chronic low back pain integrating connective tissue and nervous system mechanisms. Medical Hypothesis ; 68:74-80.

Leclaire, R., Esdaile, J.M., Suissa, S., Rossignol, M., Prouix, R., an Duouis, M, 1996 Back school in first episodes of compensated acute low back pain. A clinical trial to assess the efficacy and prevent relapse. Archives of Physical Medicine and Rehabilitation 77 7:637-639

Leibing E, Leonhardt U, Köster G, Goerlitz A, Rosenfeldt JA, Hilgers R, Ramadori G. 2002 Acupuncture treatment of chronic low-back pain a randomized, blinded, placebo-controlled trial with 9-month follow-up. Pain ; 96:189-196.

Liddle SD, Gracey JH, Baxter GD 2007. Advice for the management of low back pain: a systematic review of randomised controlled trials. Man Ther ; 12(4): $310-27$

Linton SJ 2011 Occupational Psychological factors increase the risk for back pain. A systematic review. J Occup Rehabil 11:53-56

Malasa A. Sarta G 1983 Suppression of electroacupuncture induced beta endorphin Acta endocrinol 103:469-472

Manheimer, E., White, A., Berrnan, B., Forys, K., Ernst, E. 2005. Meta analysis: Acupuncture for Low back pain. Ann Intern Med 142:651-653
Molsberger, A.F., Mau, J, Paelek, D.B., Winkler, J. 2002 Does acupuncture improve the orthopaedic management of chronic low back pain- A randomised, blinded controlled trial with three months follow up. Pain 99(3):579-87

Muthukrishnan R, Shenoy SD, Jaspal SS, Nellikunja S, Fernandes S 2010. The differential effects of core stabilization exercise regime and conventional physiotherapy regime on postural control parameters during perturbation in patients with movement and control impairment chronic low back pain. Sports Med Arthrosc Rehabil Ther Technol ; 2: 13

Pereira LM, Obara K, Dias JM, Menacho MO, Guariglia DA, Schiavoni D, Pereira HM, Cardoso JR.2011 Comparing the Pilates method with no exercise or lumbar stabilization for pain and functionality in patients with chronic low back pain Systematic review and meta-analysis. Clin Rehabil

Rosen, M.1994, Report of Clinical Standard Advisory Group Committee on Back pain HSMO, London

Sherman, K.J., Cherkin, D.C.,2002 Challenges of Acupuncture research:study design consideration, Clinical acupunct orient Med 3:200-206

Smith B, Khan I, 1988. An acupuncture program for the treatment of drug induced person Bull Narc 40: $35-41$

Sokunbi, O., Watt, P., Moore A., 2002 Changes in plasma serotonin in response to spinal stabilisation exercise in chronic low back pain patients Nig Q J Hosp Med 17(3) 108-11

van Tudler Tulder?, M.W., Cherkin, D.C., Berman, B, Lao, L, Koes, B.W. 2000. Acupuncture for low back pain.Cochrane Database Syst Rev: CD001351

Vingard E., Alexanderson K, Norlund A, 2004. Sickness presence. Scand J public Health 32:216221

Von Korff, M., Jensen, M.,P., Karoly, P. 2000 Assessing global pain severity by self report in clinical health service research Spine 25:3140-51

Zazulak B, Cholewicki J, Reeves NP 2008. Neuromuscular control of trunk stability: clinical implications for sports injury prevention. J Am Acad Orthop Surg ; 16(9): 497-505

Zuidema, H., 1985 National Statistics in the Netherlands, Ergonomics, 283 